\title{
A Prospective Examination of the Interpersonal-Psychological Theory of Suicidal Behavior Among Psychiatric Adolescent Inpatients
}

\author{
Ewa K. Czyz, MS, Johnny Berona, MS, and Cheryl A. King, PhD \\ Psychology Psychiatry, University of Michigan, Ann Arbor, MI, USA
}

\section{Abstract}

The challenge of identifying suicide risk in adolescents, and particularly among high-risk subgroups such as adolescent inpatients, calls for further study of models of suicidal behavior that could meaningfully aid in the prediction of risk. This study examined how well the InterpersonalPsychological Theory of Suicidal Behavior (IPTS) — with its constructs of thwarted belongingness (TB), perceived burdensomeness (PB), and an acquired capability (AC) for lethal self-injurypredicts suicide attempts among adolescents $(N=376) 3$ and 12 months after hospitalization. The three-way interaction between $\mathrm{PB}, \mathrm{TB}$, and $\mathrm{AC}$, defined as a history of multiple suicide attempts, was not significant. However, there were significant 2-way interaction effects, which varied by sex: girls with low $\mathrm{AC}$ and increasing $\mathrm{TB}$, and boys with high $\mathrm{AC}$ and increasing $\mathrm{PB}$, were more likely to attempt suicide at 3 months. Only high AC predicted 12-month attempts. Results suggest gender-specific associations between theory components and attempts. The time-limited effects of these associations point to TB and PB being dynamic and modifiable in high-risk populations, whereas the effects of AC are more lasting. The study also fills an important gap in existing research by examining IPTS prospectively.

Suicide is the third leading cause of death among adolescents in the United States (Centers for Disease Control \& Prevention [CDC], 2012a). Moreover, serious suicidal thoughts and one or more suicide attempts within the previous year are reported by approximately $16 \%$ and $8 \%$, respectively, of high school students, with over $2 \%$ of these students having made a suicide attempt requiring medical attention (CDC, 2012b). Severe suicidal ideation and suicide attempts are associated with psychiatric hospitalizations, distress for the youth and their families, and persisting psychosocial impairment that may extend into young adulthood (Fergusson, Horwood, Ridder, \& Beautrais, 2005; Reinherz, Tanner, Berger, Beardslee, \& Fitzmaurice, 2006; Yen et al., 2013). Identifying adolescents at elevated risk for suicidal behavior, with the goal of preventing suicide deaths and the morbidity associated with suicide attempts, is a national public health priority (U.S. Department of Health \& Human Services, 2012).

Adolescent psychiatric inpatients constitute a particularly vulnerable group, as suicidal ideation and repeated suicidal behavior persist in a considerable proportion of these youth

Address correspondence to Ewa K. Czyz, Psychology Psychiatry, University of Michigan, 4250 Plymouth Rd., Ann Arbor, MI 48109, USA; ewac@umich.edu. 
following hospitalization (Czyz \& King, 2015; Goldston et al., 1999; Prinstein et al., 2008). However, there are important gaps in the literature concerning which of these high-risk youths are most vulnerable to suicidal behavior, and how to best identify them.

The challenge of identifying suicide risk, particularly among high-risk subgroups such as adolescent inpatients, calls for further study of models of suicidal behavior that could meaningfully aid in the prediction of risk, contribute to risk formulation, and ultimately prevent youth suicide. One notable effort to understand the etiology of suicidal behavior is the Interpersonal-Psychological Theory of Suicidal Behavior (IPTS; Joiner, 2005; Van Orden et al., 2010). This theory proposes that suicidal behavior (i.e., suicide attempts and death by suicide) takes place when an individual has both the desire for death-influenced by thwarted belongingness and perceived burdensomeness - and an acquired capability to enact lethal self-injury. Thwarted belongingness involves a perception that one is alienated from others, while perceived burdensomeness is the perception that one is a burden on others. Acquired capability for suicide, however, is conceptualized as fearlessness about physical pain and death itself, which can be acquired through risky behaviors or painful and fear-provoking experiences (e.g., abuse, nonsuicidal self-injury, previous suicide attempt) that habituate a person toward suicidal action and erode the basic human instinct of selfpreservation. IPTS proposes that these three theoretical components serve as a common proximal pathway from risk factors to suicidal behavior. This framework may be particularly useful in understanding what propels some high-risk adolescents, but not others, toward taking suicidal action.

To date, few studies have examined IPTS while considering all three components simultaneously, with most studies having focused on only one or two components (e.g., Joiner et al., 2002; Van Orden, Lynam, Hollar, \& Joiner, 2006; Van Orden, Witte, Gordon, Bender, \& Joiner, 2008). In addition, little is known about the developmental appropriateness of the theory in explaining adolescent suicidal behavior. In the first study examining all components of IPTS, Joiner and colleagues (2009) demonstrated that the interaction between low belongingness, perceived burdensomeness, and acquired capability for suicide, measured by lifetime number of suicide attempts, was associated with recent suicide attempts in a clinical sample of suicidal young adults. However, two more recent studies have not found support for these three components being essential in explaining suicidal behavior. Specifically, the three-way interaction between all three constructs was not associated with suicidal history (a composite index of previous suicide attempts, frequency of suicidal ideation, previous suicidal communication, and subjective likelihood of future attempts) in a military sample of active duty airmen (Bryan, Morrow, Anestis, \& Joiner, 2010). While it is possible that this lack of support for the theory might have been influenced by the composite measure of suicidal history not sufficiently capturing suicidal behavior, a study of veterans entering inpatient psychiatric treatment revealed similar findings. In that study, the interaction between belongingness, burdensomeness, and acquired capability did not differentiate veterans with and without history of previous suicide attempts (Montheith, Menefee, Pettit, Leopoulos, \& Vincent, 2013). While methodological differences (e.g., different samples; examining recent vs. lifetime suicide attempts) might have accounted for discrepant findings, additional studies are needed to 
clarify the relationship between IPTS components and address the methodological limitations of previous research.

A key limitation of existing studies is their retrospective and cross-sectional design. These limitations preclude establishing a temporal relationship between IPTS components and suicidal behavior. A prospective study of IPTS would allow for a stronger test of the theory. Moreover, the majority of existing studies examining at least two components of IPTS have focused on adult, college, and military populations. Little is known about the applicability of the theory to an at-risk adolescent population. This is an important limitation given that the components of the theory may have differential effects on suicide risk for some developmental periods. Indeed, a recent community-based study of adults in their 20s, 40s, and 60s demonstrated that the interaction between burdensomeness and thwarted belonging was associated with increasing suicidal ideation for those in their 20s and 60s, whereas an inverse relationship was observed for those in their 40s (Christensen, Batterham, Soubelet, $\&$ Mackinnon, 2013). Although the study did not examine whether the differences across age groups were statistically significant, and replication is needed before strong conclusions can be drawn, its findings call attention to the importance of examining the relative relevance of the theory in different age groups.

It is worth noting that previous studies, although not testing IPTS directly, have provided some initial support for the relevance of the theory to adolescents. In particular, greater sense of connectedness to others has been consistently linked to a decreased likelihood of youth suicide attempts and suicidal ideation (Borowsky, Ireland, \& Resnick, 2001; Borowsky, Resnick, Ireland, \& Blum, 1999; Czyz, Liu, \& King, 2012; Eisenberg \& Resnick, 2006; Resnick et al., 1997). Less is known about the role of burdensomeness and adolescent suicide risk. One study (Woznica \& Shapiro, 1990) examined a construct that is similar to perceived burdensomeness - the "expendable child" syndrome originally proposed by Sabbath (1969). Specifically, these authors found that higher scores on a psychotherapistrated scale of expendability, or a sense of being unwanted and/or a burden on their families, differentiated adolescents with suicidal ideation or history of attempts from other adolescents (Woznica \& Shapiro, 1990). Lastly, providing first evidence about the applicability of IPTS to an adolescent population, Opperman and colleagues reported that low sense of belongingness with family combined with high perceived burdensomeness was associated with suicidal ideation among adolescents recruited from an emergency department (Opperman, Czyz, Gipson, \& King, in press). We are building on this and other existing work by testing all three IPTS components using a prospective design in a sample of acutely suicidal adolescents.

The purpose of this study is to fill important gaps in existing research by examining how well IPTS can prospectively explain suicidal behavior in a psychiatric sample of suicidal adolescents. A promising implication of studying the applicability of IPTS in this population is a more parsimonious risk assessment framework. Specifically, these teens frequently have numerous risk factors, which makes risk prediction especially difficult, while IPTS proposes a more proximal pathway from risk factors to suicidal behavior whereby individual risk factors are hypothesized to confer risk when they involve or increase likelihood that individuals will experience feelings of thwarted belongingness and perceived 
burdensomeness, and which are accompanied by acquired capability (Van Orden et al., 2010). In addition, examining the IPTS model in this sample could lead to the development of valuable intervention approaches with suicidal adolescents.

Consistent with IPTS, we hypothesized that the simultaneous presence of low sense of belonging, high perceived burdensomeness, and high acquired capability for self-injury would be associated with proximal risk of suicide attempts within 3 months of hospitalization and more distal risk within 12 months, while accounting for important correlates of suicidal behavior, such as depressive symptoms (Nock et al., 2013; Shaffer et al., 1996). We expected that the likelihood of suicide attempts would be greater in the presence of an interaction between suicidal desire (i.e., burdensomeness combined with thwarted belongingness) and acquired capability (i.e., lifetime multiple suicide attempts, conceptualized by the theory as strongly impacting acquired capability for suicide) than either component alone (see Figure 1). In addition, we examined sex as a moderator of these relationships in light of the marked gender differences in suicidal behavior, with females being more likely to attempt suicide and males being more likely to die by suicide (CDC, 2012a). The need to consider potential sex differences is particularly warranted in view of previous studies demonstrating sex-specific association between interpersonal factors such as social support - a construct similar to belongingness - and suicide-related outcomes, where significant associations were observed for female, but not male, adolescents (Kerr, Preuss, \& King, 2006; Lewinsohn, Rohde, Seeley, \& Baldwin, 2001; Mazza \& Reynolds, 1998). To the best of our knowledge, sex differences have not been examined in previous research of IPTS, an important gap that could help inform for whom the theory might have the most, or least, potential to be useful in guiding risk assessment of future suicidal behavior.

\section{METHODS}

\section{Participants}

Participants were 376 adolescents who had been psychiatrically hospitalized at a university hospital or a private hospital in the midwestern region of the United States due to acute suicidal ideation or attempt. Participants were assessed during or within 1 week of hospitalization and completed follow-up assessments 3, 6, and 12 months later. The sample was drawn from the randomized clinical trial of a social support intervention-the YouthNominated Support Team-II (YST-II)—for suicidal adolescents following hospitalization (King et al., 2009). The original study included 448 adolescents, ages 13 to 17. Please refer to King et al. (2009) for a detailed description of inclusion and exclusion criteria and study recruitment procedures. Adolescents were included in the present study if they had completed at least one follow-up assessment 3,6, or 12 months after hospitalization and for whom we had complete follow-up data on suicide attempts within 3 months and, for another set of analyses, within 12 months after hospitalization. There were no statistically significant differences between those with and without follow-up data based on key demographic characteristics, relevant clinical characteristics, or variables of interest. In addition, adolescents assigned to YST-II, versus usual care only, did not have a significantly different rate of suicide attempts within the 12-month follow-up. Participants were predominantly 
female $(72 \%)$ with a mean age of 15.59 years $(S D=1.31)$. The racial/ethnic composition of the sample was approximately $83 \%$ Caucasian, $7 \%$ African American, $0.5 \%$ Asian American/Pacific Islander, 2\% Hispanic, 0.5\% Native American, and 7\% "Other" or not identified. The annual income of adolescents' families ranged from less than $\$ 15,000$ to more than $\$ 100,000$, with the median annual family income between $\$ 40,000$ and $\$ 59,000$. Further, $10 \%$ of participants lived in families where at least one parent received public assistance.

\section{Measures}

Acquired Capability and Suicide Attempt Outcome-Lifetime multiple attempt status, assessed at baseline, and suicide attempts at each of the follow-up points were assessed with the Diagnostic Interview Schedule for Children (DISC-IV; Shaffer, Fisher, \& Lucas, 1998). The presence of suicide attempts was assessed with the question "Have you ever, in your whole life, tried to kill yourself or made a suicide attempt?" (yes/no). The time frame was adapted to capture the appropriate assessment window at each follow-up assessment. Baseline history of multiple suicide attempts was used as a measure of acquired capability, as an established measure of acquired capability (i.e., Acquired Capability for Suicide Scale; Van Orden et al., 2008) was not available. Although suicide attempt history is just one measure of acquired capability, it is perhaps the most relevant source of acquired capability, particularly in a suicidal clinical sample. Aside from being at an increased risk for subsequent suicidal behavior (Goldston et al., 1999; Hulten et al., 2001), multiple suicide attempters are also more prone to other experiences that are indicative of and lead to high acquired capability for suicide, such as higher engagement in nonsuicidal self-injury, violence, hard drug use, and being sexually or physically assaulted (Esposito, Spirito, Boergers, \& Donaldson, 2003; Rosenberg et al., 2005). Moreover, adolescents with multiple suicide attempts may also be at higher risk for lethal attempts as they more often timed their attempts so that intervention was not possible, more often reported wanting to die from their attempt, and more frequently reported regretting recovery (Miranda et al., 2008). In addition, multiple suicide attempts was used as a measure of acquired capability in a previous study of IPTS (Joiner et al., 2009), which allows for a more direct comparison of findings across studies.

Perceived Burdensomeness-Perceived burdensomeness was measured with three items from the Suicidal Ideation Questionnaire-Junior (SIQ-JR; Reynolds, 1988), a 15-item self-report questionnaire that measures frequency of a range of suicidal thoughts. The SIQJR has solid, well-documented psychometric properties (Reynolds, 1988, 1992) and has been found to predict suicidal thoughts and attempts 6 months after psychiatric hospitalization in a sample of adolescents (King, Hovey, Brand, \& Ghaziuddin, 1997). The three items include: "I thought that others would be happier if I was dead;" "I wished that I had never been born;" and "I thought that no one cared if I lived or died." Items are rated on a 7-point scale ranging from I never had this thought to almost every day. A factor analysis of the SIQ-JR based on data from two studies of inpatient adolescents (King, Woolley, Kerr, \& Vinokur, 2014) revealed that SIQ-JR has three factors, one of which (interpersonal factor) included these three items. As evidence of convergent validity, the three items correlated adequately $(0.59, p<.001)$ with six items assessing the construct of burdensomeness from 
the Interpersonal Needs Questionnaire (INQ; Van Orden, Cukrowicz, Witte, \& Joiner, 2012) in a sample of 139 12- to 15-year-olds who were recruited from a medical emergency department for participation in a larger CDC-funded intervention effectiveness trial, Links to Enhancing Teens' Connectedness, led by the third author (C. A. King). Internal consistency for the three items in this sample was 0.81 .

Thwarted Belongingness-Thwarted belongingness was measured with items from the Perceived Emotional/Personal Support Scale (PEPPS; Slavin, 1991), which has been used to measure a related construct of connectedness (Czyz et al., 2012). PEPPS assesses the degree to which respondents perceive their relationships as close, confiding, satisfying, and supportive within the domains of family, friends, and nonfamily adults. Participants listed initials of up to three people in each domain and, using a 4-point scale ranging from hardly at all to very much, rated how close they feel to each person, how satisfied they are with the help and support the person gives them, and how much they talk to each person about personal concerns. PEPPS demonstrated reasonably high test-retest reliability and internal consistency in an adolescent sample (Slavin, 1991). We focus on family belongingness because of its well-documented relationship with youth suicide attempts and suicidal ideation (e.g., Borowsky et al., 1999, 2001; Resnick et al., 1997) and because family involvement plays a key role in positive intervention effects with suicidal adolescents (Diamond et al., 2010; Esposito-Smythers, Spirito, Kahler, Hunt, \& Monti, 2011; Pineda \& Dadds, 2013), thus having most relevance in the context of this study. Each of the components/sub-scales of family belongingness (closeness, support, confiding) was averaged and used as an index of the thwarted belongingness latent variable. Scores were reverse-coded to reflect thwarted belongingness.

Depressive Symptoms-The Children's Depression Rating Scale-Revised (CDRS-R; Poznanski \& Mokros, 1996) was used to measure depressive symptoms within the previous 2 weeks. The CDRS-R is a semi-structured interview that assesses a broad range of depressive symptoms grouped into 17 areas, such as appetite disturbance, sleep disturbance, social withdrawal, or depressed feelings. Items are rated on a 5- or 7-point scale and scores range from 17 to 113 . The measure has strong psychometric properties in studies with adolescents (e.g., Emslie et al., 1997; Shain, Naylor, \& Alessi, 1990). Interinterviewer reliability for total scores, assessed prior to data collection, was high (mean alpha across raters of .98).

\section{Data Analysis}

Structural equation modeling (SEM) was used to examine two IPT models predicting suicide attempts within 3 and 12 months after hospitalization. Analyses were conducted using Mplus, version 6.1 (Byrne, 2012). The models included two latent variables (thwarted belongingness and perceived burdensomeness, with three indicators each) and an observed acquired capability variable measured by lifetime multiple attempts (yes/no). Confirmatory factor analysis was first conducted to determine whether the selected thwarted belongingness and perceived burdensomeness indicators adequately represented these latent variables (measurement model described in Results). Next, SEM was used to examine relationships between the latent and measured variables. Main effects of thwarted belongingness, 
perceived burdensomeness, and acquired capability on the suicide outcome were examined first. Two-way interactions between these variables were considered next. The three-way interaction between thwarted belongingness, perceived burdensomeness, and acquired capability was examined as the last step. In addition, multigroup analyses were conducted to examine whether these relationships varied depending on sex, and Wald tests of parameter equality constraints were used to examine the significance of any sex differences. All analyses controlled for baseline depressive symptoms. In addition, given that the original sample came from an intervention study, the intervention group was included as a covariate; however, as it was not a significant predictor and results did not vary with it in the models, it was removed from the final models.

\section{RESULTS}

\section{Characteristics of the Sample}

Prior to baseline, 91 (24\%) adolescents had not attempted suicide, 135 (36\%) had made one attempt, and $150(40 \%)$ had a history of two or more attempts. Sex did not differentiate baseline multiple suicide attempters from nonmultiple attempters. Twenty-eight adolescents, or approximately $7 \%$ of the sample, made at least one suicide attempt at the 3-month followup, and 64 (17\%) made at least one suicide attempt during the 1-year follow-up. Adolescents with multiple attempt histories were more likely to attempt suicide (24\% vs. $14 \%$ ), $\chi^{2}$ (1, N $=355)=4.81, p=.03$ prior to 12 months, but not prior to 3 months after hospitalization. Sex did not differentiate suicide attempters from nonattempters during the follow-up. The correlations among all study measures are included in Table 1.

\section{Measurement Model}

The results of the confirmatory factor analysis showed good model fit based on Comparative Fit Index (CFI) and Tucker-Lewis Index (TLI) of 0.99 each, and the root-mean squarederror of approximation (RMSEA) of $0.02(90 \% \mathrm{CI}=0.001,0.07)$. In general, CFI and TLI values of above 0.90 and RMSEA values of 0.05 to as high as 0.08 indicate good model fit to the data (Byrne, 2012). The standardized factor loadings for the perceived burdensomeness latent variable were high $(0.84,0.68,0.80 ; p<.001)$, as were loadings for the thwarted belongingness variable $(0.88,0.79,0.68 ; p<.001)$, indicating that the selected items captured the two latent variables.

\section{Predicting Suicide Attempts Using Structural Equation Models}

Suicide Attempts 3 months After Hospitalization-The interaction between thwarted belongingness, perceived burdensomeness, and acquired capability did not significantly predict suicide attempts 3 months after hospitalization (Table 2). The interaction between thwarted belongingness and perceived burdensomeness was also not significant. Similarly, neither thwarted belongingness, perceived burdensomeness, nor acquired capability, when examined as main effects, predicted suicide attempts within 3 months of hospitalization. However, the model yielded two significant two-way interaction effects, which were moderated by sex. 
First, the interaction between thwarted belongingness and acquired capability had overall significance for the entire sample $(B=0.85, p=.04)$, where adolescents with low acquired capability (i.e., non-multiple suicide attempters) and higher levels of thwarted belongingness were more likely to attempt suicide $[B=0.78 ;(\mathrm{OR}=2.18,95 \% \mathrm{CI}=1.10,4.33) ; p=.02]$ when compared with those with high acquired capability (i.e., multiple suicide attempters) $[B=-0.07 ;(\mathrm{OR}=0.93,95 \% \mathrm{CI}=0.63,1.38) ; p=.73]$. Examination of sex differences revealed that the interaction between low acquired capability and higher levels of thwarted belongingness was significant for girls $(B=1.22, p=.02)$, but not boys $(B=-0.22, p=.71)$, suggesting that this relationship was mostly influenced by, and more salient for, girls.

However, it is important to note that the group moderation effect did not reach significance ( $t$ $=3.24, p=.07)$.

Second, there was a significant interaction between perceived burdensomeness and acquired capability, but only when moderated by $\operatorname{sex}(t=7.43, p=.006)$; this interaction was not significant for the overall sample $(-0.48, p=.28)$. Specifically, the interaction between acquired capability and perceived burdensomeness was significant for boys $(B=-2.66, p=$. $007)$, but not girls $(B=0.36, p=.49)$. An exploration of this interaction showed that, relative to boys with high acquired capability, boys with low acquired capability and higher levels of perceived burdensomeness were less likely to attempt suicide 3 months posthospitalization $[B=-1.38,(\mathrm{OR}=0.25,95 \% \mathrm{CI}=0.73,0.86) ; p=.03]$. A graphic summary of the relationship between IPTS components and the attempt outcome 3 months after hospitalization is provided in Figure 1.

Suicide Attempts Within 12 Months After Hospitalization-The interaction between thwarted belongingness, perceived burdensomeness, and acquired capability also did not predict suicide attempts 12 months after hospitalization (Table 3). Similarly, neither thwarted belongingness nor perceived burdensomeness, when examined as main effects, predicted suicide attempts 12 months after hospitalization. In addition, and unlike observed for suicide attempts within 3 months, there were no significant 2-way interactions. The only significant predictor of suicide attempts during the 12-month period was acquired capability, where adolescents with high acquired capability were more likely to attempt suicide $[B=$ $0.32,(\mathrm{OR}=1.38,95 \% \mathrm{CI}=1.01,1.88) ; p=.04]$, even after controlling for important covariates. This significant main effect was not moderated by $\operatorname{sex}(t=0.19, p=.66)$.

\section{Supplemental Analyses with Peer-Thwarted Belongingness}

As mentioned in the methods section, the focus of this study, in examining the relevance of IPTS to adolescents, was on the construct of thwarted belongingness within the family. However, because the results only partially supported the theory, we re-ran all the analyses with peer-thwarted belongingness (note: the peer-thwarted belongingness latent variable was also based on the three belongingness indexes derived from the PEPPS; Slavin, 1991). The analytic approach was the same as described earlier. The measurement model with peerthwarted belongingness indicated a good model fit $[\mathrm{CFI}=0.99$; TLI $=0.99 ; \mathrm{RMSEA}=0.03$ $(90 \% \mathrm{CI}=0.001,0.07)]$. The standardized factor loadings for the peer-thwarted belongingness variable were high $(0.86,0.81,0.76 ; p<.001)$, indicating that the selected items captured the latent variable. The pattern of these supplemental results was similar to 
the results reported for family-thwarted belongingness. Specifically, the three-way interaction between peer-thwarted belongingness, perceived burdensomeness, and acquired capability did not significantly predict suicide attempts 3 or 12 months after hospitalization. In addition, the model predicting suicide attempts at 3 months yielded a similar pattern of two-way interactions moderated by sex. The interaction between perceived burdensomeness and acquired capability was again moderated by $\operatorname{sex}(t=9.90, p=.002)$, indicating that this interaction was significant only for boys $(B=-2.25, p=.002)$. Boys with low acquired capability and higher levels of perceived burdensomeness were less likely to attempt suicide 3 months posthospitalization $(B=-1.38, p=.03)$, while an opposite pattern was found for boys with high acquired capability and higher levels of perceived burdensomeness. The thwarted belongingness by acquired capability interaction was moderated by sex $(t=3.91, p$ $<.05)$, whereby girls with low acquired capability and higher levels of peer-thwarted belongingness were more likely to attempt suicide at 3 months ( $B=0.56, p=.06)$; however, this finding did not reach statistical significance. As was the case in the analysis involving family thwarted belongingness, only high acquired capability was associated with 12-month suicide attempts $(B=0.29, p=.04)$.

\section{DISCUSSION}

Seeking to fill important gaps in the literature, in this study we examined how well the ITPS predicts suicide attempts in a large sample of psychiatrically hospitalized suicidal adolescents within 3 and 12 months after hospitalization, and whether or not the relationship between the theory's components and suicide attempts varied by sex. To our knowledge, this is the first study to test IPTS prospectively, to include a high-risk sample of suicidal adolescents, and to consider sex as a moderator. Our findings offer partial support for the theory among suicidal adolescents and highlight the importance of considering sex differences in applying the theory to suicidal behavior.

A primary finding was that, contrary to the hypothesis, the interaction between thwarted belongingness, perceived burdensomeness, and acquired capability did not predict suicide attempts in the more immediate risk period of 3 months or the more distal period of 12 months after hospitalization. Although surprising and contrary to the theory, this finding is consistent with two retrospective studies of military populations (Bryan et al., 2010; Montheith et al., 2013), neither of which found support for the three components being essential in explaining previous suicide attempts or suicidal history. Thus far, only one study has demonstrated that the simultaneous presence of all three components was associated with suicidal behavior (recent suicide attempts; Joiner et al., 2009). Together with Bryan and colleagues and Montheith and colleagues, our findings raise questions about the relative importance of, and necessity for, the three IPTS constructs in explaining suicidal behavior, and underscore the need for additional prospective research of IPTS to further clarify the discrepancies between theory and research. In particular, because our study relied on proxy measures of theory constructs (e.g., multiple attempt history as an indicator of high acquired capability), studies using established measures of theory constructs are needed to provide more confidence in the findings. 
Another key finding in our study concerns sex-specific associations between the different theory components and suicide attempt risk. Boys with low acquired capability and higher levels of perceived burdensomeness were at lower risk of suicide attempts within 3 months of hospitalization compared with boys with high acquired capability; this effect did not hold for girls. It is notable that previous studies have similarly reported an interaction between acquired capability and perceived burdensomeness in the absence of a significant interaction between all three components (Bryan et al., 2010; Montheith et al., 2013). The replication of this pattern of findings in an adolescent population suggests that the role of perceived burdensomeness in the presence of high acquired capability constitutes a clinically significant combination in high-risk populations. However, our study is the first to show that this relationship varies depending on sex. The finding that burdensomeness combined with high acquired capability predicted suicide attempts among boys, but not girls, is noteworthy because of the difficulty in identifying clinically valuable risk factors for suicidal behavior among high-risk adolescent males (King, Jiang, Czyz, \& Kerr, 2014).

While more research is needed to better understand the mechanisms accounting for this finding, one possible reason for the significant interaction among suicidal adolescent boys, but not girls, may be due to these boys' greater reactivity to feelings of burdensomeness, which might have been exacerbated by the recent hospitalization. In a recent study of hospitalized adolescents, experiences of heightened negative affect sensitivity or reactivity were more strongly associated with elevated suicidal ideation for males (Selby, Yen, \& Spirito, 2013). It is thus possible that the males in this sample experienced a lower threshold for suicidal action in response to an emotional trigger such as feelings of burdensomeness. The fact that this vulnerability was observed among boys with multiple suicide attempt history is also consistent with previous research showing that adolescents with multiple suicide attempt history have higher levels of affect dysregulation (Esposito et al., 2003). Another possible explanation might have to do with gender differences in attitudes toward the self. A recent study found that among men, but not among women, depressive symptoms conferred elevated risk for suicide in the presence of higher value placed on autonomy (Bamonti, Price, \& Fiske, 2014). Although attitudes toward autonomy were not assessed in our study, adolescence is a transitional period in which sense of autonomy is developing. The sense of perceived burdensomeness may capture similar constructs that are potentially more salient for males than females. This study provides initial evidence that burdensomeness feelings may warrant particular attention among adolescent males, although additional studies are needed to replicate and more fully understand its significance.

The second sex-specific association found in the study was between thwarted belongingness and acquired capability. Although low acquired capability accompanied by higher levels of thwarted belongingness was associated with an overall suicide attempt risk within 3 months of hospitalization, this relationship appeared to be mostly influenced by girls. However, some caution is warranted in interpreting this finding due to the group moderation effect not reaching statistical significance. It is important to note that we may have been insufficiently powered to detect significant, particularly more modest, effects with our modeling approach (MacCallum, Browne, \& Sugawara, 1996). Nevertheless, the interaction between low acquired capability and higher levels of thwarted belongingness had a significant robust effect among girls and was nonsignificant for boys, suggesting that the overall interaction 
effect was largely driven by girls. This is consistent with previous studies showing that family support is more salient for girls in predicting less severe suicide-related outcomes, including suicidal ideation and attempts (Kerr et al., 2006; Lewinsohn, Rohde, Seeley, \& Baldwin, 2001). Others have shown that the protective effect of family connectedness extends to both male and female adolescents (Ackard, Neumark-Sztainer, Story, \& Perry, 2006; Borowsky et al., 2001; Czyz \& King, 2015; Eisenberg \& Resnick, 2006). Future research is needed to add to our understanding of sex differences in IPTS.

Some attention is warranted to the interaction between acquired capability and thwarted belongingness having had an opposite than expected effect on post-hospitalization suicide attempt risk. A possible explanation for this finding might be related to the way we measured acquired capability and the dynamic nature of thwarted belongingness within high-risk families across time. Our previous work has shown that adolescents low in acquired capability (single and nonattempters) experienced greater sense of belongingness with family 3 months after hospitalization relative to the high acquired capability group (multiple attempters), even though both groups reported similar levels of belongingness with family at hospitalization (Czyz \& King, 2015). This indicates greater variability in thwarted belongingness for adolescents with low acquired capability during the 3-month period and a potential floor effect among adolescents with high acquired capability. In this context, our findings indicating that thwarted belongingness is a risk factor for those with low acquired capability — or that decreasing thwarted belongingness may be protective in this groupseem more conceivable, although inconsistent with IPTS. However, it is worth noting that the unexpected interaction effect was previously reported in a study of veterans entering inpatient psychiatric treatment (Montheith et al., 2013), suggesting that methodology alone is unlikely to explain the contradictory findings. In the Montheith and colleagues' study, higher levels of thwarted belongingness were associated with lower likelihood of previous multiple suicide attempts among veterans with low acquired capability, while high acquired capability accompanied by greater thwarted belongingness was not associated with multiple attempt history (L. Montheith, personal communication, October 28, 2013). Moreover, in yet another military study, the interaction between thwarted belongingness and acquired capability was not significantly associated with suicidal history (Bryan et al., 2010). While the explicit focus of IPTS is on the simultaneous effect of all its three components, that the combined effect of thwarted belongingness and high acquired capability does not appear to impact risk of suicidal behavior in this and other studies is of significance. First, it could explain why the interaction between all three components was not supported, beyond any methodological differences across studies. Second, it invites consideration of perceived burdensomeness in combination with acquired capability as the more prominent components of the theory.

Finally, our study points to time-varying impact of IPTS components on suicide attempt risk after psychiatric hospitalization. Specifically, perceived burdensomeness combined with acquired capability was associated with suicide attempts within the first 3 months of hospitalization, but no longer impacted risk of suicide attempts within the entire 12-month follow-up. A similar time-limited effect was observed for thwarted belongingness combined with acquired capability. The only significant predictor of suicide attempt within 12 months of hospitalization was high acquired capability. Together, this pattern of findings suggest that 
thwarted belongingness and perceived burdensomeness are more dynamic (Van Orden et al., 2010) and represent more state-dependent influence on suicide risk. By the same token, these factors might be modifiable in high-risk populations, and thus could be of value in development of intervention approaches for suicidal adolescents. In fact, there is emerging evidence for the value of increasing inpatient adolescents' sense of connectedness shortly after hospitalization to improve suicide-related outcomes, including suicidal ideation, depressive symptoms, and suicide attempts (Czyz et al., 2012). That multiple suicide attempt history had the most lasting effect on suicide attempt risk is consistent with acquired capability being conceptualized as the static component of ITPS (Van Orden et al., 2010), and is in line with previous research demonstrating its strong association with future suicidal behavior (Goldston et al., 1999; Hulten et al., 2001). This underscores the importance of prevention of the onset and maintenance of behaviors and experiences that increase acquired capability (e.g., initiation of suicidal behavior, transition from one-time to multiple attempt status).

\section{Study Limitations}

This study has several important limitations. The generalizability of our findings is limited by the largely female and Caucasian sample from one region of the United States. In addition, because participants were psychiatrically hospitalized due to a suicide risk concern, the range of the variables might be reduced and also findings may not apply to adolescents from outpatient clinics or from the community. However, the study's focus on hospitalized suicidal adolescents who are particularly vulnerable to suicidal crises is also its strength. The study also has measurement limitations related to our use of proxy measures for some of the theory constructs. Specifically, while our use of multiple suicide attempts represents one of the strongest indicators of acquired capability and is theoretically sound (due to its association with subsequent suicidal behavior and other indicators of acquired capability such as nonsuicidal self-injury, violence, hard drug use, sexual and physical assault), the strength of our conclusions is tempered by these measurement limitations. Future work would be strengthened by use of measures specifically designed to assess IPTS constructs (e.g., Van Orden et al., 2008) and by consideration of various indicators of acquired capability (e.g., nonsuicidal self-injury, abuse history, physical injuries). For example, this might help solidify if the results are influenced by the underlying construct of acquired capability as conceptualized by the theory (high pain tolerance, fearlessness about death itself) rather than another construct associated with multiple suicide attempts (e.g., emotion dysregulation; Esposito et al., 2003). However, it is noteworthy that the results of this study are consistent with a previous study that utilized the measures specifically established to measure IPTS (Montheith et al., 2013), thus providing some confidence in our results not being an artifact of construct measurement. In addition, the pattern of results was similar when we examined thwarted belongingness in the context of family and, in supplemental analysis, peer relationships. Despite these limitations, this study has important strengths, including a large sample of acutely suicidal adolescents and a prospective design that allowed for a stronger test of IPTS. 


\section{Implications and Conclusions}

The current study has important implications for intervening with suicidal adolescents at risk for suicide attempts after hospitalization. It is noteworthy that thwarted belongingness, perceived burdensomeness, and depressive symptoms were not independently associated with suicide attempts; only acquired capability predicted suicide attempts, but not within the first 3 months of hospitalization. In contrast, the combined effect of these predictors proved to be more robust in differentiating suicide attempters from nonattempters, particularly in the critical period shortly after hospitalization. The importance of considering the synergistic effect of multiple risk factors is consistent with IPTS and might be especially relevant for assessment and intervention with high-risk adolescent groups. In particular, asking adolescent boys about thoughts of feeling like a burden on others, especially if they report high acquired capability for suicidal behavior, could help identify those at highest risk for subsequent suicidal behavior. Assessing thoughts of burdensomeness among adolescent males has added value because identifying high-risk boys is difficult, even when incorporating known risk factors such as suicidal ideation (King, Jiang, et al., 2014). Our findings also indicate that improving hospitalized adolescents', particularly girls', sense of belongingness might reduce risk of suicidal behavior shortly after hospitalization; however, this benefit appears to extend to those with low acquired capability for suicidal behavior, which highlights the need to strengthen belongingness among adolescents with multiple suicide attempt histories whose social support systems might be considerably weaker (Cohen-Sandler, Berman, \& King, 1982; Kotila \& Lonnqvist, 1987). In addition, our findings reveal that it is critical to provide ongoing suicide risk assessment after hospitalization as the effect of acquired capability persists over time. Conversely, the more dynamic nature of thwarted belongingness and perceived burdensomeness highlights an opportunity for intervention (e.g., challenge burdensomeness-related cognitions; encourage activities that will lessen sense of burdensomeness; help promote close and supportive relationships within the family). The gender-specific pathways for suicidal behavior provide useful information about tailoring intervention in this high-risk population.

Our findings also have implications for future IPTS research. Consistent with previous studies (Bryan et al., 2010; Montheith et al., 2013), our findings call into question the concept that all three IPTS components are essential in predicting suicidal behavior. Specifically, we suggest that the absence or contradictory effect of thwarted belongingness —when combined with perceived burdensomeness or acquired capability—is likely accounting for the lack of support for the theory and as such should be reconsidered as being its essential component. At the same time, we provide additional support for perceived burdensomeness as playing a more prominent role in IPTS. Our findings similarly suggest that the presence of gender-specific pathways might also account for why all three components, when considered simultaneously, do not appear to be essential in predicting suicide attempts. Future work is needed to replicate this pattern of findings prospectively (particularly using established measures of theory constructs and/or more inclusive indicators of acquired capability), in both adolescent and other samples, to provide more confidence in these results and help inform future theoretical advances. Our study stresses the need to consider gender-specific differences in future IPTS research. Finally, the timelimited effect of ITPS constructs in this study suggests that future longitudinal research 
would be strengthened by utilizing a more fine-grained analysis of these constructs at frequent assessment time points to more closely study their dynamic influence on suicidal behavior. Such a fine-grained analysis might be particularly relevant to high-risk samples, including suicidal hospitalized populations, who may experience more shifts in thwarted belongingness and perceived burdensomeness in response to suicidal crises across time.

\section{References}

Ackard DM, Neumark-Sztainer D, Story M, Perry C. Parent-child connectedness and behavioral and emotional health among adolescents. American Journal of Preventive Medicine. 2006; 30:59-66. [PubMed: 16414425]

Bamonti PM, Price EC, Fiske A. Depressive symptoms and suicide risk in older adults: Value placed on autonomy as a moderator for men but not women. Suicide and Life-Threatening Behavior. 2014; 44:188-199. [PubMed: 24851257]

Borowsky IW, Ireland M, Resnick MD. Adolescent suicide attempts: Risks and protectors. Pediatrics. 2001; 107:485-493. [PubMed: 11230587]

Borowsky IW, Resnick MD, Ireland M, Blum RW. Suicide attempts among American Indian and Alaska Native youth: Risk and protective factors. Archives of Pediatrics and Adolescent Medicine. 1999; 153:573-580. [PubMed: 10357296]

Bryan CJ, Morrow CE, Anestis MD, Joiner TE. A preliminary test of the interpersonal-psycholgical theory of suicidal behavior in a military sample. Personality and Individual Differences. 2010; 48:347-350.

Byrne, BM. Structural equation modeling with Mplus: Basic concepts, applications, and programming. New York: Routledge; 2012.

Centers for Disease Control and Prevention. Web-based injury statistics query and reporting system (WISQARS). National Center for Injury Prevention and Control; 2012a. Retrieved September 19, 2013, from http://www.cdc.gov/injury/wisqars/index.html

Centers for Disease Control and Prevention. Youth Risk Behavior Surveilance-United States, 2011. Morbidity and Mortality Weekly Report. 2012b; 61(4)

Christensen H, Batterham PJ, Soubelet A, Mackinnon AJ. A test of the interpersonal theory of suicide in a large community-based cohort. Journal of Affective Disorders. 2013; 144:225-234. [PubMed: 22862889]

Cohen-Sandler R, Berman AL, King RA. A follow-up study of hospitalized suicidal children. Journal of the American Academy of Child and Adolescent Psychiatry. 1982; 21:398-403.

Czyz EK, King CA. Longitudinal trajectories of suicidal ideation and subsequent suicide attempts among adolescent inpatients. Journal of Clinical Child and Adolescent Psychology. 2015; 44:181193. [PubMed: 24079705]

Czyz EK, Liu Z, King CA. Social connectedness and 1-year trajectories among suicidal adolescents following psychiatric hospitalization. Journal of Clinical Child and Adolescent Psychology. 2012; 41:214-226. [PubMed: 22417194]

Diamond GS, Wintersteen MB, Brown GK, Diamond GM, Gallop R, Shelef K, et al. Attachmentbased family therapy for adolescents with suicidal ideation: A randomized controlled trial. Journal of the American Academy of Child and Adolescent Psychiatry. 2010; 49:122-131. [PubMed: 20215934]

Eisenberg ME, Resnick MD. Suicidality among gay, lesbian, and bisexual youth: The role of protective factors. Journal of Adolescent Health. 2006; 39:662-668. [PubMed: 17046502]

Emslie GJ, Rush AJ, Weinberg WA, Kowatch RA, Hughes CW, Carmody T, et al. A double-blind, randomized, placebo-controlled trial of fluoxetine in children and adolescents with depression. Archives of General Psychiatry. 1997; 54:1031-1037. [PubMed: 9366660]

Esposito C, Spirito A, Boergers J, Donaldson D. Affective, behavioral, and cognitive functioning in adolescents with multiple suicide attempts. Suicide and Life-Threatening Behavior. 2003; 33:389399. [PubMed: 14695054] 
Esposito-Smythers C, Spirito A, Kahler CW, Hunt J, Monti P. Treatment of co-occurring substance abuse and suicidality among adolescents: A randomized trial. Journal of Consulting and Clinical Psychology. 2011; 79:728-739. [PubMed: 22004303]

Fergusson DM, Horwood LJ, Ridder EM, Beautrais AL. Suicidal behaviour in adolescence and subsequent mental health outcomes in young adulthood. Psychological Medicine. 2005; 35:983993. [PubMed: 16045065]

Goldston DB, Daniel SS, Reboussin DM, Reboussin BA, Frazier PH, Kelley AE. Suicide attempts among formerly hospitalized adolescents: A prospective naturalistic study of risk during the first 5 years after discharge. Journal of the American Academy of Child and Adolescent Psychiatry. 1999; 38:660-671. [PubMed: 10361783]

Hulten A, Jiang GX, Wasserman D, Hawton K, Hjelmeland H, De Leo D, et al. Repetition of attempted suicide among teenagers in Europe: Frequency, timing and risk factors. European Child and Adolescent Psychiatry. 2001; 10:161-169. [PubMed: 11596816]

Joiner, T. Why people die by suicide. Cambridge: Harvard University Press; 2005.

Joiner TE, Pettit JW, Walker RL, Voelz ZR, Cruz J, Rudd MD, et al. Perceived burdensomeness and suicidality: Two studies on the suicide notes of those attempting and those completing suicide. Journal of Social and Clinical Psychology. 2002; 21:531-545.

Joiner TE, Van Orden KA, Witte TK, Selby EA, Ribeiro JD, Lewis R, et al. Main predictions of the interpersonal-psychological theory of suicidal behavior: Empirical tests in two samples of young adults. Journal of Abnormal Psychology. 2009; 118:634-646. [PubMed: 19685959]

Kerr DCR, Preuss LJ, King CA. Suicidal adolescents' social support from family and peers: Genderspecific associations with psychopathology. Journal of Abnormal Child Psychology. 2006; 34:99_ 110 .

King CA, Hovey JS, Brand E, Ghaziuddin N. Prediction of positive outcomes for adolescent psychiatric inpatients. Journal of the American Academy of Child and Adolescent Psychiatry. 1997; 36:1434-1442. [PubMed: 9334557]

King CA, Jiang Q, Czyz EK, Kerr DCR. Suicidal ideation of psychiatrically hospitalized adolescents has one-year predictive validity for suicide attempts in girls only. Jounral of Abnormal Child Psychology. 2014; 42:467-477.

King CA, Kerr DC, Passarelli MN, Foster CE, Merchant CR. One-year follow-up of suicidal adolescents: Parental history of mental health problems and time to post-hospitalization attempt. Journal of Youth and Adolescence. 2010; 39:219-232. [PubMed: 19967398]

King CA, Klaus N, Kramer A, Venkataraman S, Quinlan P, Gillespie B. The Youth-Nominated Support Team-Version II for suicidal adolescents: Randomized controlled intervention trial. Journal of Consulting and Clinical Psychology. 2009; 77:880-893. [PubMed: 19803568]

King CA, Woolley ME, Kerr DCR, Vinokur A. Factor structure, internal consistency, and predictive validity of the Suicidal Ideation Questionnaire-Junior (SIQ-JR) in a sample of suicidal adolescents. 2014 Unpublished manuscript.

Kotila L, Lonnqvist J. Adolescents who make suicide attempts repeatedly. Acta Psychiatrica Scandinavica. 1987; 76:386-393. [PubMed: 3425365]

Lewinsohn PM, Rohde P, Seeley JR, Baldwin CL. Gender differences in suicide attempts from adolescence to young adulthood. Journal of the American Academy of Child and Adolescent Psychiatry. 2001; 40:427-434. [PubMed: 11314568]

MacCallum RC, Browne MW, Sugawara HM. Power analysis and determination of sample size for covariance structure modeling. Psychological Methods. 1996; 1:130-149.

Mazza JJ, Reynolds WM. A longitudinal investigation of depression, hopelessness, social support, and major and minor life events and their relation to suicidal ideation in adolescents. Suicide and LifeThreatening Behavior. 1998; 28:358-374. [PubMed: 9894304]

Miranda R, Scott M, Hicks R, Wilcox HC, Munfakh JLH, Shaffer D. Suicide attempt characteristics, diagnoses, and future attempts: Comparing multiple attempters to single attempters and ideators. Journal of the American Academy of Child and Adolescent Psychiatry. 2008; 47:32-40. [PubMed: 18174823] 
Montheith LL, Menefee DS, Pettit JW, Leopoulos WL, Vincent JP. Examining the interpersonalpsychological theory of suicide in an inpatient veteran sample. Suicide and Life-Threatening Behavior. 2013; 43:418-428. [PubMed: 23556542]

Nock MK, Green JG, Hwang I, McLaughlin KA, Sampson NA, Zaslavsky AM, et al. Prevalence, correlates, and treatment of lifetime suicidal behavior among adolescents: Results From the National Comorbidity Survey Replication Adolescent Supplement. JAMA. 2013; 70:300-310.

Opperman K, Czyz EK, Gipson PY, King CA. Connectedness and perceived burdensomeness in adolescents at elevated suicide risk: An examination of the interpersonal theory of suicidal behavior. Archives of Suicide Research. In press.

Pineda J, Dadds MR. Family intervention for adolescents with suicidal behavior: A randomized controlled trial and mediation analysis. Journal of the American Academy of Child and Adolescent Psychiatry. 2013; 52:851-862. [PubMed: 23880495]

Poznanski, EO.; Mokros, HB. Children's Depression Rating Scale-Revised (CDRS-R). Los Angeles, CA: Western Psychological Services; 1996.

Prinstein MJ, Nock MK, Simon V, Aikins JW, Cheah CS, Spirito A. Longitudinal trajectories and predictors of adolescent suicidal ideation and attempts following inpatient hospitalization. Journal of Consulting and Clinical Psychology. 2008; 76:92-103. [PubMed: 18229987]

Reinherz HZ, Tanner JL, Berger SR, Beardslee WR, Fitzmaurice GM. Adolescent suicidal ideation as predictive of psychopathology, suicidal behavior, and compromised functioning at age 30 . American Journal of Psychiatry. 2006; 163:1226-1232. [PubMed: 16816228]

Resnick MD, Bearman PS, Blum RW, Bauman KE, Harris KM, Jones J, et al. Protecting adolescents from harm: Findings from a national longitudinal study of adolescent health. Journal of the American Medical Association. 1997; 278:823-832. [PubMed: 9293990]

Reynolds, WM. Suicidal Ideation Questionnaire: Professional manual. Odessa, FL: Psychological Assessment Resources; 1988.

Reynolds, WM. Measurement of suicidal ideation in adolescents. Paper presented at the annual meeting of the American Association of Suicidology; Chicago, IL. 1992.

Rosenberg HJ, Jankowski MK, Sengupta A, Wolfe RS, Wolford GL, Rosenberg SD. Single and multiple suicide attempts and associated health risk factors in New Hampshire adolescents. Suicide and Life-Threatening Behavior. 2005; 35:547-557. [PubMed: 16268771]

Sabbath JC. The suicidal adolescent: The expendable child. Journal of the American Academy of Child Psychiatry. 1969; 8:272-285. [PubMed: 5782320]

Selby EA, Yen S, Spirito A. Time-varying prediction of thoughts of death and suicidal ideaiton in adolescents: Weekly ratings over 6-month follow-up. Journal of Clinical Child and Adolescent Psychology. 2013; 42:481-495. [PubMed: 23148530]

Shaffer, D.; Fisher, P.; Lucas, C. NIMH DISC Editorial Board. DISC-IV. Diagnostic Interview Schedule for Children (Youth informant and parent informant interviews): Epidemiologic version. New York: Joy and William Ruane Center to Identify and Treat Mood Disorders, Division of Child Psychiatry, Columbia University; 1998.

Shaffer D, Gould MS, Fisher P, Trautman P, Moreau D, Kleinman M, et al. Psychiatric diagnosis in child and adolescent suicide. Archives of General Psychiatry. 1996; 53:339-348. [PubMed: 8634012]

Shain BN, Naylor M, Alessi N. Comparison of self-rated and clinician-rated measures of depression in adolescents. American Journal of Psychiatry. 1990; 147:793-795. [PubMed: 2343926]

Slavin LA. Validation studies of the PEPSS, a measure of perceived emotional support for use with adolescents. Journal of Adolescent Research. 1991; 6:316-335.

U.S. Department of Health and Human Services, Office of the Surgeon General and National Action Alliance for Suicide Prevention. National strategy for suicide prevention: Goals and objectives for action. Washington, DC: Author; 2012.

Van Orden KA, Cukrowicz KC, Witte TK, Joiner TE Jr. Thwarted belongingness and perceived burdensomeness: Construct validity and psychometric properties of the Interpersonal Needs Questionnaire. Psychological Assessment. 2012; 24:197-215. [PubMed: 21928908]

Van Orden KA, Lynam ME, Hollar D, Joiner TE Jr. Perceived burdensomeness as an indicator of suicidal symptoms. Cognitive Therapy and Research. 2006; 30:457-467. 
Van Orden KA, Witte TK, Cukrowicz KC, Braithwaite SR, Selby EA, Joiner TE Jr. The interpersonal theory of suicide. Psychological Review. 2010; 117:575-600. [PubMed: 20438238]

Van Orden KA, Witte TK, Gordon KH, Bender TW, Joiner TE Jr. Suicidal desire and the capability for suicide: Tests of the interpersonal-psychological theory of suicidal behavior among adults. Journal of Consulting and Clinical Psychology. 2008; 76:72-83. [PubMed: 18229985]

Woznica JG, Shapiro JR. An analysis of adolescent suicide attempts: The expendable child. Journal of Pediatric Psychology. 1990; 15:789-796. [PubMed: 2283581]

Yen S, Weinstock LM, Andover ES, Sheets E, Selby EA, Spirito A. Prospective predictors of adolescent suicidality: 6 month post hospitalization follow-up. Psychological Medicine. 2013; 43:983-993. [PubMed: 22932393] 


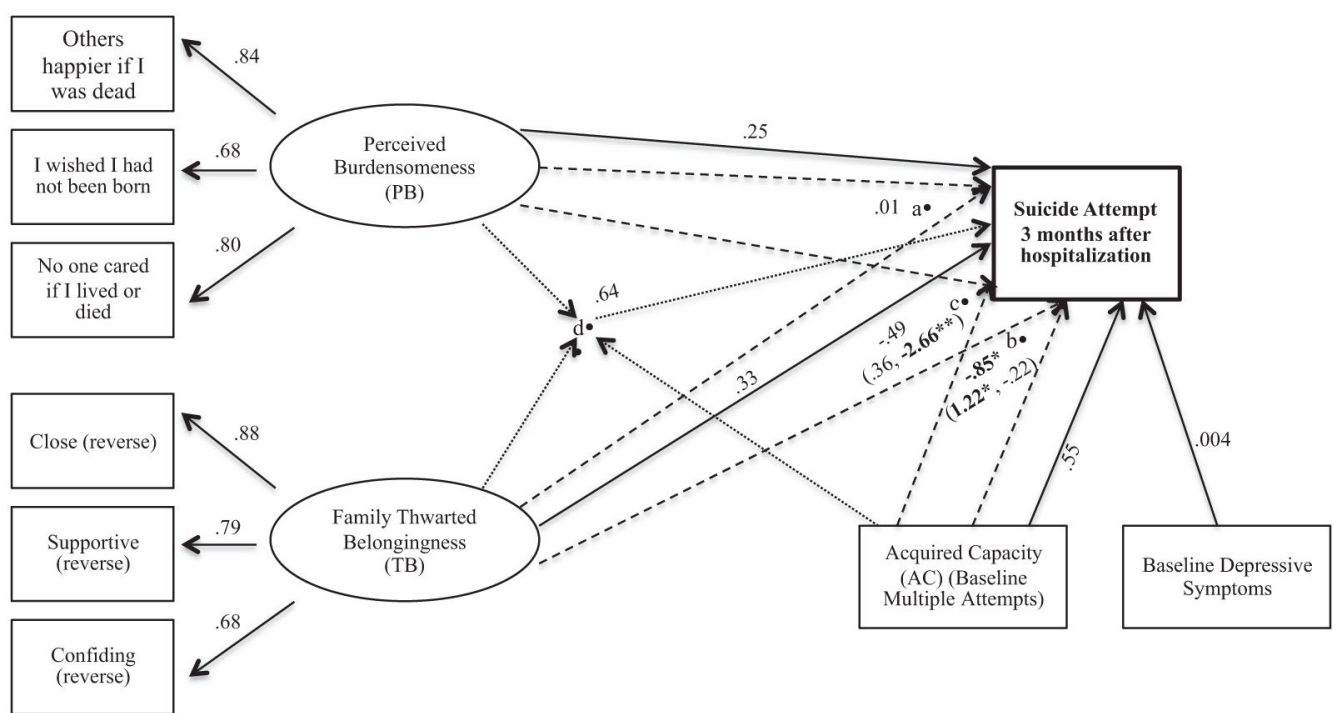

Figure 1. Structural equation model of the Interpersonal-Psychological Theory of suicidal behavior predicting suicide attempts 3 months after hospitalization

Notes: 1. Solid lines indicate main effects (Model 1); 2. Dashed lines indicate 2-way interactions where $\mathrm{a}=\mathrm{TBxPB}($ Model 2), $\mathrm{b}=\mathrm{TBxAC}($ Model 3), $\mathrm{c}=\mathrm{PBxAC}($ Model 4); 3. Dotted line indicates a 3-way interaction $d=$ TBxPBxAC (Model 5); 4. Significantly moderated effects are shown in parentheses for (girls, boys); 5. * $p<.05, * * p<.01 ; \mathbf{6}$. All factor loadings for PB and TB are significant at $p<.001$. 


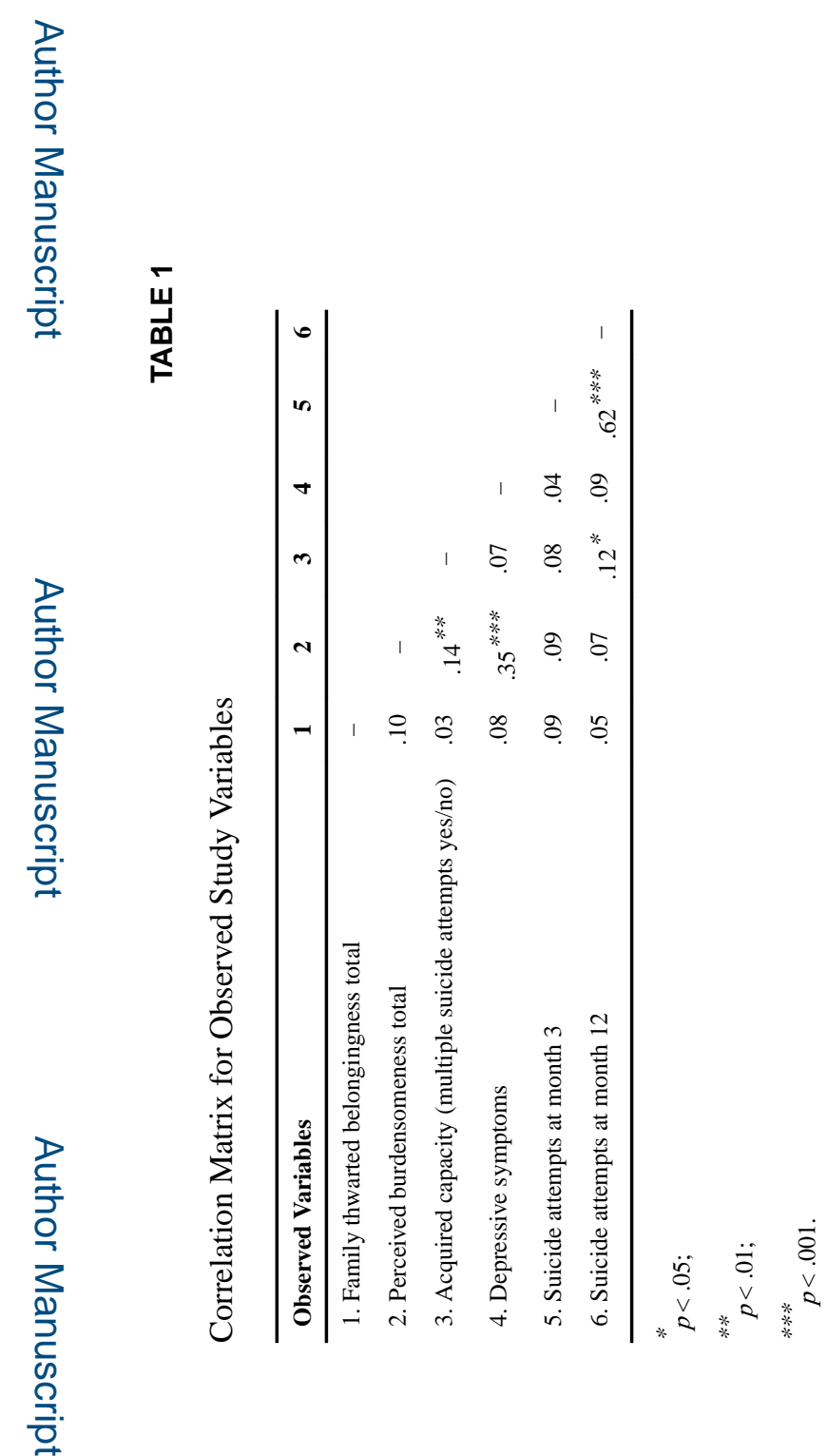

J Relig Health. Author manuscript; available in PMC 2017 August 01. 


\section{를}

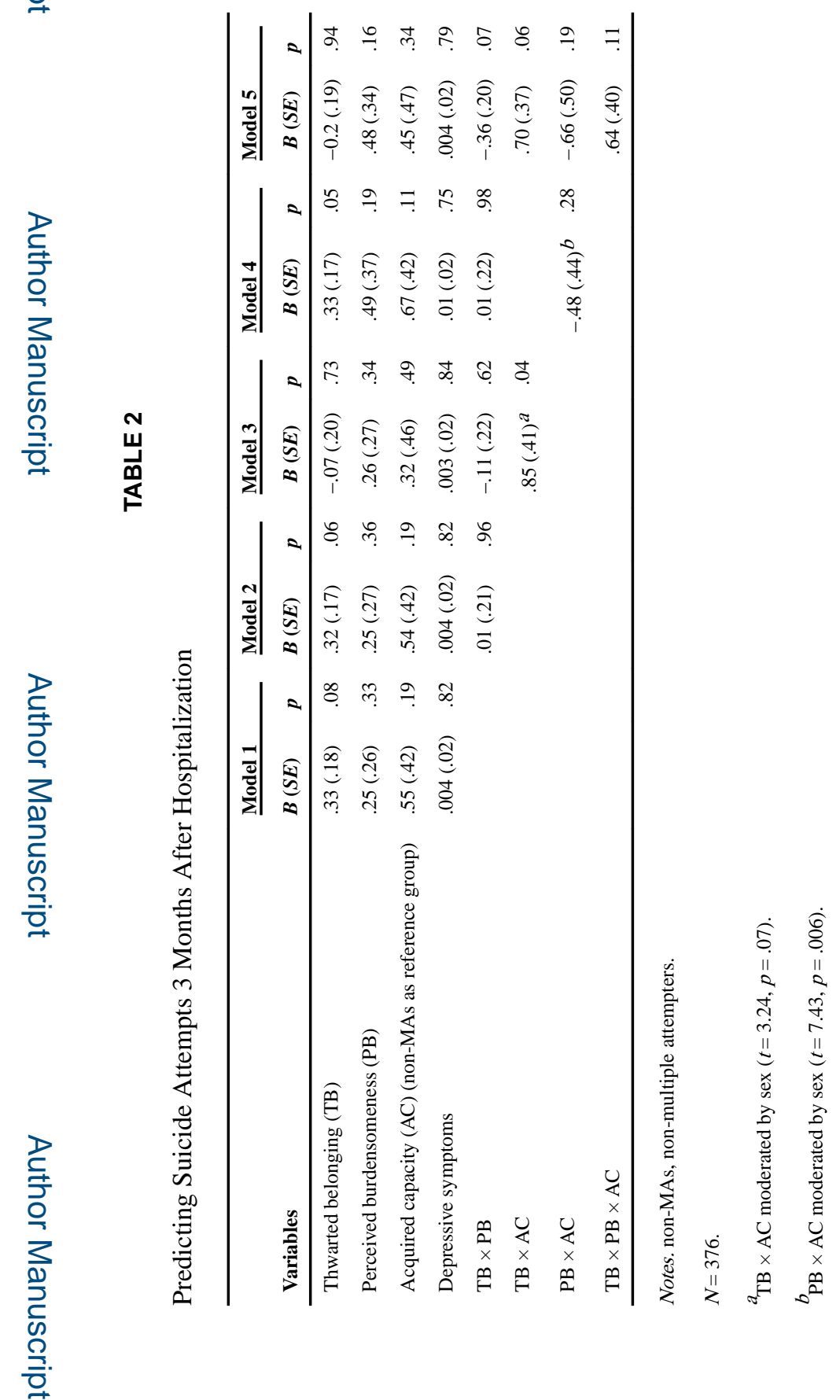

J Relig Health. Author manuscript; available in PMC 2017 August 01. 


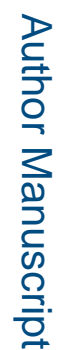

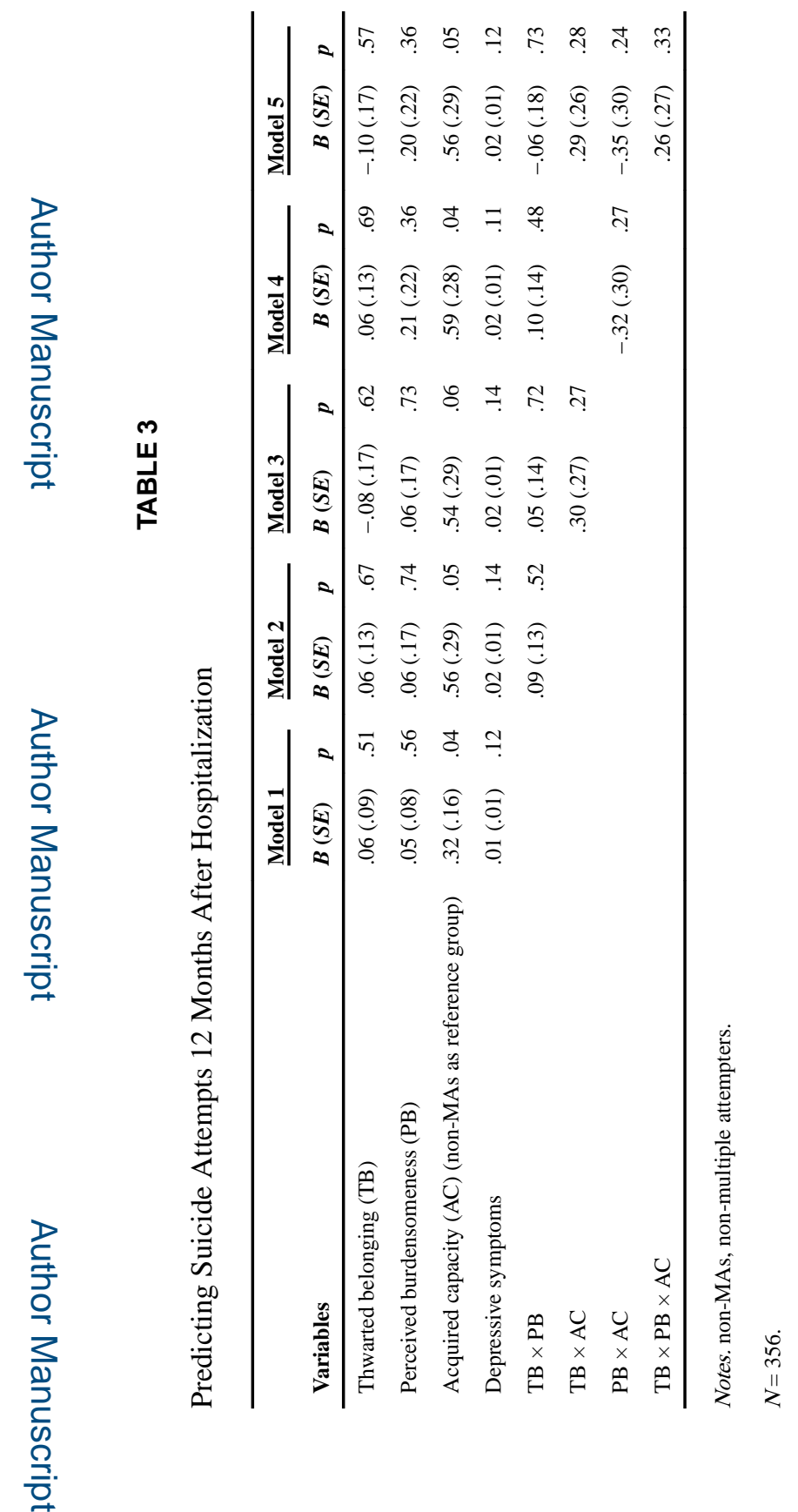

J Relig Health. Author manuscript; available in PMC 2017 August 01. 